\title{
Pauli equation for BPS monopoles in moduli space
}

\author{
P.P. Purohit ${ }^{*}$, V.P. Pandey and B.S. Rajput \\ Department of Physics, Kumaun University, Nainital-263 002, India
}

\begin{abstract}
Undertaking the study of behaviour of a BPS monopole moving in the field of another BPS monopole, in moduli space, Dirac's equation has been solved for energy eigen values and it has been shown that spin momentum of an interacting BPS monopole behaves as extra energy source. Introducing suitable spinors, the Pauli equation for a BPS monopole moving in the field of another BPS monopole has been solved in moduli space and it has been shown that adhoc introduction of spin in the system of two BPS monopoles perceptibly modifies the energy eigen values and eigen functions of bound states of the system.
\end{abstract}

\section{INTRODUCTION}

Apart from 't Hooft-Polyakov ${ }^{(1,2)}$ non-Abelian monopole Bogomol'nyi-PrasadSommerfield (BPS) monopoles ${ }^{(3)}$ have attracted much attention in a variety of contexts in theoretical and mathematical physics ${ }^{(4)}$ and more recently in pure mathematics BPS monopoles provide a three-dimensional example of topological solitons of Bogomol'nyi type; they are static, finite energy solutions of classical field equations and stable because their energy attains a lower topological bound, the Bogomol'nyi bound. Much progress in understanding of the dynamics of such solitons has been made over the past decade using the idea of moduli space approximation ${ }^{(5)}$. In this scheme one approximates the slow motion of several interacting solitons by geodesic motion on the Riemanian manifold of static multi-soliton solutions. To quantise, one works in the Schrodinger picture: wave functions are complex valued functions on the moduli space and the quantum Hamiltonian is taken to be proportional to the Laplace-Beltrami operator ${ }^{(6)}$. In general the mathematical analysis of moduli space is still very difficult problem, but in the case of two BPS monopoles one could exploit special properties, in particular the hyperkahler property of the metric, and obtained sufficient information about the moduli

\footnotetext{
* Corresponding author
} 
space to calculate geodesics and to study the Laplace operator in some detail. The analysis of the moduli space is summarised in the recent book by Atiyah and Hitchin ${ }^{(7)}$ and the paper of N. Seiberg ${ }^{(8)}$. Jackiw and Rebbi first observed that the Dirac operator coupled to a t' Hooft-Polyakov monopole has zero modes ${ }^{(9)}$ and gave a physical interpretation of them; later Callias ${ }^{(10)}$ rigorously proved an index theorem for the Dirac operator in the background of a general BPS (multi-) monopole. Undertaking the study of monopoles and dyons in moduli space we ${ }^{(11)}$ have analyzed the extended structure of non-Abelian dyons and obtained the structure for dyonic mass and electric and magnetic fields in the interior region of dyon and showed that when collective coordinates of monopoles are time dependent it acquires momentum and electric charge and become a moving dyon. We have also analyzed the connection of moduli space of monopoles with normalized bosonic and fermionic zero modes ${ }^{(11)}$. We have undertaken the study of kinematics and monodromies around singularities in quantum moduli space of fourdimensional $\mathrm{N}=2$ supersymmetric theory and obtained spectra of BPS state in weak and strong coupling regions ${ }^{(12)}$. In the present paper, we have undertaken the study of Pauli equation for BPS monopoles in moduli space and showed that the interaction of spin and the potential leads to an extra-energy expressible in terms of spin momentum of the particle concerned. Analyzing Dirac equation in moduli space, the study of interaction of spin and orbital angular momentum has been undertaken. We have also undertaken the study of a BPS monopole moving in the field of another BPS monopole by introducing suitable spinors and bound states energy eigen values and eigen functions have been obtained in moduli space.

\section{BEHAVIOUR OF A BPS MONOPOLE IN THE FIELD OF ANOTHER BPS MONOPOLE}

Our standard references for background material on BPS monopoles will be the book by Atiah and Hitchin ${ }^{(7)}$ and the paper by Gibbons and Manton ${ }^{(6)}$. We follow the notational conventions adopted there and denote by $A_{i}, i=1,2,3$, the cartesian components of an SU(2) gauge potential $\vec{A}$ on $R^{3}$ and by $\Phi$ a Higgs field in the adjoint representation of $S U(2)$. More specifically, writing su(2) for the Lie algebra of $S U(2)$, we have four maps

$A_{i}, \Phi: R^{3} \rightarrow S U(2)$.

We use the gauge potential to define the covariant derivative

$D_{i}=\partial_{i}+g A_{i}$

where $g$ is the magnetic charge of BPS monopole and the curvature is given by

$F_{i j}=\partial_{i} A_{j}-\partial_{j} A_{i}+\left[A_{i}, A_{j}\right]$. 
We shall often refer to the gauge group $\mathrm{SU}(2)$ as the isospin group and use a basis $\mathrm{T}_{\mathrm{a}}$ (a $=1,2,3$,) of su(2) satisfying $\left[T_{a}, T_{b}\right]=\in_{a b c} T_{C}$, which can be expressed in terms of the Pauli matrices $\tau_{a}$ via $T_{a}=\frac{1}{2 i} \tau_{a}$. We also require a norm \|\| on su(2) which we take to be $\|T\|^{2}=-\frac{1}{2} \operatorname{tr} T^{2}$. We can then introduce the space $A$ of pairs $(\vec{A}, \Phi)$ which satisfy the boundary condition

$\lim _{|\vec{x}| \rightarrow \infty}\|\Phi(\vec{x})\|=1$

and the base point condition

$\lim _{x_{3} \rightarrow \infty} \Phi\left(0,0, x_{3}\right)=-T_{3}$.

The group $G$ of gauge transformations is, by definition, the space of maps $g: R^{3} \rightarrow S U(2)$ which satisfy the base point condition

$\lim _{3} \rightarrow \infty$

$\mathrm{G}$ acts freely on $A$ and dividing by the action, we obtain the true configuration space

$\wp=\frac{A}{\mathrm{G}}$.

Finally; we introduce a non-Abelian magnetic field

$B_{i}=\frac{1}{2} \in_{i j k} F_{j k}$

and define that a BPS monopole is a pair $(\vec{A}, \Phi) \in A$ which satisfy the Bogomol'nyi equation

$B_{i}=D_{i} \Phi$.

We introduce the notation $\mathrm{X}_{\mathrm{k}}$ for the space of all k-monopoles. $\mathrm{X}_{\mathrm{k}}$ is an infinite dimensional manifold on which the group $G$ acts freely. Quotienting $X_{k}$ by this action, we obtain the moduli space $\mathrm{M}_{\mathrm{k}}$ which is differentiable manifold of dimension $4 \mathrm{k}$. It has a natural Riemannian metric introduced from the Yang-Mills-Higgs (YMH) kinetic energy functional and it has been explained ${ }^{(7)}$ why $M_{k}$ equipped with this metric is a hyperkahler manifold. Since $\mathrm{M}_{\mathrm{k}}$ is precisely the subset of $\wp$ on which the potential energy functional $\mathrm{V}$ is minimal for given $\mathrm{k}$, it is a natural candidate for the configuration space of a truncation of the YMH theory in the topological sector with winding number $\mathrm{k}$. Manton et. $\mathrm{al}^{(4)}$ have given the interpretation of the $4 \mathrm{k}$ parameters and the moduli space of a single monopole. $\mathrm{M}_{1}$ is a flat manifold of the form

$M_{1}=R^{3} \times S^{1}$

with $\mathrm{R}^{3}$ and $\mathrm{S}^{1}$ as position and angular coordinates respectively. 
Writing $\psi(\mathrm{t}, \overrightarrow{\mathrm{x}})$ for a four-component spinor which also transforms under the fundamental representation of the $\mathrm{SU}(2)$ isospin group, the (1+4)-dimensional Dirac equation in the temporal gauge $\mathrm{A}_{0}=0$, may be written as $\left[-\left\{\Gamma^{0} \otimes \partial_{t}+c \Gamma^{\mu} \otimes D_{\mu}\right\}+m_{1} c^{2}\right] \psi=0$,

where $m_{1}$ is the mass of BPS monopole while $D_{i}$ is defined by equation (4.2) and $D_{4}=\Phi$. Specifically, we consider $\mathrm{SU}(2)$ gauge potential $A_{\mu}, \mu=1,2,3,4$ on $R^{4}=R^{3} \times R$ which are independent of $x_{4}$ and have self-dual curvature

$F_{k \lambda}=\frac{1}{2} \in_{k \lambda \mu \nu} F_{\mu \nu}$.

It may be checked that this equation is equivalent to the Bogomol'nyi equation if the fourth component of the gauge potential is identified with the Higgs field, $\mathrm{A}_{4}=\Phi$. We can obtain five $4 \times 4$ complex matrices $\left(\Gamma^{o}, \Gamma^{\mu}\right)$, from the standard Dirac $\gamma$-matrices:

$\Gamma^{0}=\gamma^{0}, \Gamma^{i}=\gamma^{i}, \Gamma^{4}=-i \gamma^{5}=\gamma^{0} \gamma^{1} \gamma^{2} \gamma^{3}$.

$\psi(\mathrm{t}, \overrightarrow{\mathrm{x}})$ really transforms under a spinor representation of $\mathrm{SO}(1,4)$ but we can think of it as an S0 $(1,3)$ spinor by restricting to the Lorentz transformations in $\mathrm{SO}(1,3) \subset \mathrm{SO}(1,4)$ respecting the condition $\mathrm{x}_{4}=0$. Dirac's equation for a BPS monopole moving in an external field of another BPS monopole may then be written as $\left[-\left\{\Gamma^{0} \otimes \partial_{t}+c \Gamma^{\mu} \otimes\left(\partial_{\mu}+g A_{\mu}\right)\right\}+m_{1} c^{2}\right] \psi=0$.

Multiplying by $\Gamma^{4}$, we get

$\left[-\Gamma^{4} \gamma^{0} \otimes \partial_{t}-c \Gamma^{4} \gamma^{i} \otimes D_{i}-c\left(\Gamma^{4}\right)^{2} \otimes\left(\partial_{4}+g A_{\mu}\right)+\Gamma^{4} m_{1} c^{2}\right] \psi=0$.

Using $\Gamma^{4}=1_{4}, D_{4}=\Phi=c\left(\partial_{4}+g A_{4}\right)$ and using equation (9), we get from equation (11):

$\left[-\left(\begin{array}{cc}1_{2} & 0 \\ 0 & -1_{2}\end{array}\right) \otimes \partial_{t}-C\left(\begin{array}{cc}0 & \sigma_{i} \\ -\sigma_{i} & 0\end{array}\right) \otimes D_{i}-\left(\begin{array}{cc}1_{2} & 0 \\ 0 & 1_{2}\end{array}\right) \otimes \Phi+\left(\begin{array}{cc}1_{2} & 0 \\ 0 & 1_{2}\end{array}\right) m_{1} c^{2}\right] \psi=0$

or

$i \hbar \frac{\partial}{\partial}\left(\begin{array}{cc}1_{2} & 0 \\ 0 & -1_{2}\end{array}\right) \psi=\left[-c\left(\begin{array}{cc}0 & \sigma_{i} \otimes D_{i} \\ -\sigma_{i} \otimes D_{i} & 0\end{array}\right)-\left(\begin{array}{cc}1_{2} \otimes \Phi & 0 \\ 0 & 1_{2} \otimes \Phi\end{array}\right)+\left(\begin{array}{cc}1_{2} & 0 \\ 0 & 1_{2}\end{array}\right) m_{1} c^{2}\right] \psi$.

The relativistic energy of the particle includes also its rest energy $\mathrm{m}_{1} \mathrm{c}^{2}$. This must be excluded in arriving at the non-relativistic approximation and we therefore replace $\psi$ by a function $\psi$ ' defined as follows:

$\psi=\psi^{\prime} e^{-i m_{1} c^{2} t / \hbar}$. 
Then from equation (12), we have

$$
\begin{gathered}
{\left[i \hbar \frac{\partial}{\partial t}+m_{1} c^{2}\right]\left(\begin{array}{cc}
1_{2} & 0 \\
0 & -1_{2}
\end{array}\right) \psi^{\prime}=\left[-c\left(\begin{array}{cc}
0 & \sigma_{i} \otimes D_{i} \\
-\sigma_{i} \otimes D_{i} & 0
\end{array}\right)-\left(\begin{array}{cc}
1_{2} \otimes \Phi & 0 \\
0 & 1_{2} \otimes \Phi
\end{array}\right)\right.} \\
\left.+\left(\begin{array}{cc}
1_{2} & 0 \\
0 & 1_{2}
\end{array}\right) m_{1} c^{2}\right] \psi^{\prime} .
\end{gathered}
$$

Substituting $\psi^{\prime}=\left(\begin{array}{l}\xi \\ \eta\end{array}\right)$, where $\xi$ and $\eta$ are two-component functions, we obtain $\left[\begin{array}{c}\left(i \hbar \frac{\partial}{\partial}+m_{1} c^{2}\right) \xi \\ -\left(i \hbar \frac{\partial}{\partial}+m_{1} c^{2}\right) \eta\end{array}\right]=\left(\begin{array}{cc}-c & \sigma_{i} \otimes D_{i} \eta \\ c & \sigma_{i} \otimes D_{i} \xi\end{array}\right)+\left(\begin{array}{l}-1_{2} \otimes \Phi \xi \\ -1_{2} \otimes \Phi \eta\end{array}\right)+\left(\begin{array}{cc}m_{1} c^{2} & \xi \\ m_{1} c^{2} & \eta\end{array}\right)$.

From equation (13) we get

$$
\begin{aligned}
& {\left[i \hbar \frac{\partial}{\partial}+1_{2} \otimes \Phi\right] \xi=-c \sigma_{i} \otimes\left(\partial_{i}+g A_{i}\right) \eta} \\
& -\left[i \hbar \frac{\partial}{\partial}-1_{2} \otimes \Phi+2 m_{1} c^{2}\right] \eta=-c \sigma_{i} \otimes\left(\partial_{i}+g A_{i}\right) \xi .
\end{aligned}
$$

In the first approximation, only the term $2 m_{1} c^{2} \eta$ is retained on the left hand side of (15), which gives

$$
\eta=-\frac{1}{2 m_{1} c} \sigma_{i} \otimes\left(\partial_{i}+g A_{i}\right) \xi
$$

Substitution of (16) in to (14) gives

$$
\begin{aligned}
i \hbar \frac{\partial \xi}{\partial} & =\left[\frac{1}{2 m_{1}}\left(\vec{p}_{i}+g A_{i}\right)^{2}-1_{2} \otimes \Phi-\frac{g \hbar}{2 m_{1}} \sigma_{i} \otimes \text { curl } A_{i}\right] \xi \\
& =\hat{H} \xi
\end{aligned}
$$

where $\partial_{i}=\vec{p}_{i}$ is the momentum of BPS monopole.

This is Pauli equation for BPS monopoles in moduli space. It has the following extra spin contribution in the energy gained by BPS monopole while moving in the field of another BPS monopole;

$$
E^{\prime}=-\frac{g \hbar}{2 m_{1}}\left(\sigma_{i} \otimes \operatorname{curl} A_{i}\right)
$$

This can also be written as

$$
E^{\prime}=\mu_{g} \otimes \operatorname{curl} A_{i}=-\mu_{g^{\prime}}\left(\sigma_{i} \otimes \operatorname{curl} A_{i}\right)
$$

where

$$
\mu_{g^{\prime}}=\frac{g \hbar}{2 m_{1}}
$$

is defined as Bohr magneton for the system and 


$$
\mu_{g}=\mu_{g^{\prime}} \sigma_{i}
$$

as spin moment of BPS monopole. Consequently, extra-energy term in the Hamiltonian may be interpreted as the energy of interaction of the spin-moment of a BPS monopole with a vector field, resulting from the space rotation of four-potential $A_{\mu}^{a}$. The third component of the spin moment operator for BPS monopole may be written as:

$\left(\mu_{g}\right)_{3}=\frac{g \hbar}{2 m_{1}} \sigma_{3}$

the eigen values of which are

$\pm \frac{g \hbar}{2 m_{1}}= \pm \mu_{g^{\prime}}$

\section{SPIN-ORBIT INTERACTION}

Let us consider the motion of a BPS monopole in the field of another BPS monopole retaining terms of up to those of order $v^{2} / c^{2}$. Substituting $A_{i}=0$ and $\mathrm{E}=\mathrm{i} \hbar \frac{\partial}{\partial \mathrm{t}}$ in equations (14) and (15), we find

$\left[E+1_{2} \otimes \Phi\right]_{j}=-c \sigma_{i} \otimes \vec{p}_{i} \eta$

$-\left[E-1_{2} \otimes \Phi+2 m_{1} c^{2}\right] \eta=c \sigma_{i} \otimes \vec{p}_{i} \xi$.

We calculate from (25) the function $\eta$ up to terms of first order in $\left(E-1_{2} \otimes \Phi\right) / 2 m_{1} c^{2}$. Substituting the value $\eta=-\frac{1}{2 m_{1} c}\left[1-\frac{E-1_{2} \otimes \Phi}{2 m_{1} c^{2}}\right]\left(\sigma_{i} \otimes \vec{p}_{i}\right) \xi$

in to equation (24), we find an equation containing only one two-component function:

$\left(E+1_{2} \otimes \Phi\right) \xi=-\frac{1}{2 m_{1}}\left(\sigma_{i} \otimes \vec{p}_{i}\right)\left[1-\frac{E-1_{2} \otimes \Phi}{2 m_{1} c^{2}}\right]\left(\sigma_{i} \otimes \vec{p}_{i}\right) \xi$

which on simplification gives the following expression for energy operator (Hamiltonian) in the first approximation;

$$
\begin{aligned}
\hat{H}=\frac{1}{2 m_{1}}\left[1-\frac{E-1_{2} \otimes \Phi}{2 m_{1} c^{2}}\right] \vec{p}_{i}^{2}-1_{2} \otimes \Phi-\frac{i \hbar}{4 m_{1}^{2} c^{2}}\left[\vec{\nabla}\left(1_{2} \otimes \Phi\right) \otimes \vec{p}_{i}\right] \\
\left.+\frac{\hbar}{4 m_{1}^{2} c^{2}}\left[\sigma_{i} \otimes \hat{\nabla}\left(1_{2} \otimes \Phi\right) \otimes \vec{p}_{i}\right\}\right] .
\end{aligned}
$$

The expression for Hamiltonian, in second approximation, can be derived by using another function

$$
\chi=\hat{u} \xi
$$


the normalization of which up to second order leads to the following value of factor $\hat{u}$ $\hat{u} \approx 1-\frac{\vec{p}_{i}^{2}}{8 m_{1}^{2} c^{2}}$

Using this value of $\hat{u}$ (and hence of $\chi$ ), we get the following relativistic expression for corresponding Hamiltonian up to terms of order $\mathrm{v}^{2} / \mathrm{c}^{2}$;

$$
\begin{aligned}
& \hat{H}= {\left[1+\frac{\vec{p}_{i}^{2}}{8 m_{1}^{2} c^{2}}\right] \hat{H}\left[1-\frac{\vec{p}_{i}^{2}}{8 m_{1}^{2} c^{2}}\right] } \\
&= {\left[\frac{\vec{p}_{i}^{2}}{2 m_{1}}-1_{2} \otimes \Phi\right]+\left[\frac{\hbar^{2}}{8 m_{1}^{2} c^{2}} \vec{\nabla}^{2}\left(1_{2} \otimes \Phi\right)\right]-\left[\frac{\left(E-1_{2} \otimes \Phi\right)^{2}}{2 m_{1} c^{2}}\right] } \\
&+\left[\frac{\hbar}{4 m_{1}^{2} c^{2}}\left[\sigma_{i} \otimes\left\{\nabla\left(1_{2} \otimes \Phi\right) \times \vec{p}_{i}\right)\right]\right. \\
&=\hat{H}_{0}+\hat{H}_{1}+\hat{H}_{2}+\hat{H}_{3}=\hat{H}_{0}+\hat{H}_{I}
\end{aligned}
$$

where $\hat{H}_{O}$ corresponds to the non-relativistic term of the Hamiltonian, while $\hat{H}_{I}$ is the relativistic term to the Hamiltonian various parts of which arise due to different relativistic interaction, The quantity $\hat{H}_{1}$ is called contact interaction operator, analogous to the term introduced by Darwin ${ }^{(13)}$ for electronic case. $\hat{H}_{2}$ is the relativistic correction term due to the dependence of kinetic energy on momentum. Finallly,

$$
\hat{H}_{3}=\frac{\hbar\left[\sigma_{1} \otimes\left\{\vec{\nabla}\left(1_{2} \otimes \Phi\right) \times \vec{p}_{i}\right\}\right]}{4 m_{1}^{2} c^{2}}
$$

is the so-colled spin-orbit interaction operator.

In a spherically symmetric field

$$
\vec{\nabla} \Phi=\frac{\vec{r}}{r} \frac{d \Phi}{d r} \text {. }
$$

Substituting this expression in to equation (29), we find the spin-orbit interaction operator for the motion of a spin-1/2 particle in a spherically symmetric field:

$$
\hat{H}_{3}=\frac{d}{d r}\left(1_{2} \otimes \Phi\right) \frac{(\hat{S} \otimes \hat{L})}{2 m_{1}^{2} c^{2} r},
$$

where $\hat{L}=\vec{r} \times \vec{p}_{i}$ is the orbital angular momentum operator and $\hat{S}=\frac{1}{2} \hbar \sigma_{i}$ is the spin angular momentum operator. For $\Phi=-\frac{g^{2}}{r}$, equation (30) reduces to

$$
\hat{H}_{3}=\frac{g^{2}}{2 m_{1}^{2} c^{2} r^{3}} 1_{2} \otimes(\hat{S} \otimes \hat{L})
$$


which clearly demonstrates that, besides the contribution of Higgs field, the interaction of spin and orbital angular momenta of moving BPS monopole also contributes to the energy operator.

\section{PAULI EQUATION FOR A BPS MONOPOLE IN THE FIELD OF ANOTHER BPS MONOPOLE IN MODULI SPACE}

For analyzing the motion of BPS monopole in the field of another BPS monopole with the inclusion of spin effect, let us start with the following Schrodinger equation of a spinning BPS monopole in the field of another BPS monopole in moduli space;

$\left[-\frac{1}{2 m_{1}} \hat{\nabla}^{2}+1_{4} \otimes \Phi(r)+F(r) \hat{L} \otimes \sigma_{i}\right]\left[\begin{array}{l}\psi_{1} \\ \psi_{2}\end{array}\right]=E\left[\begin{array}{l}\psi_{1} \\ \psi_{2}\end{array}\right]$

where $\hat{\nabla}$ is $\left(\vec{p}_{i}+g A_{i}\right)$ and spin-orbit interaction $F(r) \hat{L} \otimes \sigma_{i}$ will be treated as small perturbation.

The unperturbed Hamiltonian

$\hat{H}_{0}=-\frac{1}{2 m_{1}} \hat{\nabla}^{2}+1_{4} \otimes \Phi(r)$

where Higgs potential

$\Phi(r)=-\frac{g^{2}}{r}$

represents the familiar central force problem for the system of a BPS monopole moving in the field of another BPS monopole and the spin-orbit interaction energy $\mathrm{H}$ 'is given by

$$
\hat{H}^{\prime}=\frac{g^{2}}{2 m_{1}^{2} c^{2}}\left(\frac{1}{r^{3}}\right) 1_{2} \otimes \hat{S} \otimes \hat{L} .
$$

To simplify the above equation, we introduce the total angular momentum as $\hat{J} \otimes \hat{J}=\hat{L} \otimes \hat{L}+\hat{S} \otimes \hat{S}+2 \hat{L} \otimes \hat{S}$.

So the Pauli operator for $\hat{H}^{\prime}$ is given by

$\left(\hat{H}^{\prime}\right)_{P}=\frac{g^{2}}{4 m_{1}^{2} c^{2}}\left\langle\frac{1}{r^{3}}\right)_{2} \otimes\left[(\hat{J} \otimes \hat{J})_{P}-(\hat{L} \otimes \hat{L})_{p}-(\hat{S} \otimes \hat{S})_{p}\right]$.

Thus the Pauli wave equation becomes

$$
(\hat{H})_{P} \psi_{P}=\left\lfloor\left(\hat{H}_{0}\right)_{P}+\left(\hat{H}^{\prime}\right)_{P}\right\rfloor \psi_{P}=W \psi_{P}
$$

where

$$
\left(\hat{H}_{0}\right)_{P}=\left(\begin{array}{cc}
\hat{H}_{O} & 0 \\
0 & \hat{H}_{0}
\end{array}\right)_{P}=\left(\begin{array}{cc}
-\frac{1}{2 m_{1}} \hat{\nabla}^{2}-\frac{g^{2}}{r} 1_{4} & 0 \\
0 & -\frac{1}{2 m_{1}} \hat{\nabla}^{2}-\frac{g^{2}}{r} 1_{4}
\end{array}\right)_{P}
$$


and

$$
\psi_{P}=\left(\begin{array}{l}
\psi_{+} \\
\psi_{-}
\end{array}\right)_{P}
$$

represents the Pauli wave function. The Pauli wave equation for unperturbed Hamiltonian is given as

$$
\begin{aligned}
& \left(\begin{array}{cc}
\hat{H}_{0} & 0 \\
0 & \hat{H}_{0}
\end{array}\right)\left(\begin{array}{l}
\psi_{+}(0) \\
\psi_{-}(0)
\end{array}\right)=W(0)\left(\begin{array}{l}
\psi_{+}(0) \\
\psi_{-}(0)
\end{array}\right) \\
& \text { or } \\
& \hat{H}_{0} \psi_{ \pm}(0)=W(0) \psi_{ \pm}(0)
\end{aligned}
$$

This equation can be solved by the method of separation of variables by writing the wave function as

$$
\psi=\frac{U(r)}{r} Y_{g, l, m}(\theta, \phi)
$$

where $Y_{g, l, m}(\theta, \phi)$ is independent of $\mathrm{r}$ (it may be treated as angular function) and the radial function $\left[\frac{U(r)}{r}\right]=R(r)$ satisfy the equation

$$
r^{2}\left\{\frac{1}{r R(r)} \frac{d^{2}}{d r^{2}}(r R)+2 m_{1}\left(E-1_{4} \otimes \Phi\right)\right\}=-\frac{\Lambda Y_{g, l, m}(\theta, \phi)}{Y_{g, l, m}(\theta, \phi)}
$$

with

$$
\Lambda=\frac{1}{\operatorname{Sin} \theta} \frac{\partial}{\partial \theta}\left(\operatorname{Sin} \theta \frac{\partial}{\partial \theta}\right)+\frac{1}{\operatorname{Sin}^{2} \theta} \frac{\partial^{2}}{\partial \phi^{2}}
$$

and $\Phi$ is defined by equation (32a). Substituting the value of $\Phi$ from equation (32a) in to equation (41), we get

$\frac{1}{r^{2}} \frac{d}{d r}\left[r^{2} \frac{d R}{d r}\right]+2 m_{1}\left[E+\frac{g^{2} 1_{4}}{r}-\frac{l(l+1)}{2 m_{1} r^{2}}\right] R(r)=0$.

We introduce the dimensionless variable

$$
\rho=\alpha r
$$

so that equation (43) becomes

$$
\frac{1}{\rho^{2}} \frac{d}{d \rho}\left[\rho^{2} \frac{d R}{d \rho}\right]+\left[\frac{2 m_{1} E}{\alpha^{2}}+\frac{2 m_{1} g^{2} 1_{4}}{\alpha \rho}-\frac{l(l+1)}{\rho^{2}}\right] R(\rho)=0
$$

where $\alpha^{2}=8 m_{1}|E|=-8 m_{1} E$.

Equation (43a ) may be written as

$$
\frac{1}{\rho^{2}} \frac{d}{d \rho}\left(\rho^{2} \frac{d R}{d \rho}\right)+\left[\frac{\lambda}{\rho}-\frac{1}{4}-\frac{l(l+1)}{\rho^{2}}\right] R(\rho)=0
$$


where

$\lambda=g^{2} 14 \sqrt{\frac{m_{1}}{-2 E}}$

Equation (44) yields the following energy eigen value for the system of a BPS monopole spinning around another BPS monopole;

$E_{n}=-\frac{m_{1} g^{4} 1_{4}}{n^{2}}$

where $\mathrm{n}=0,1,2, \ldots$ and $\psi_{ \pm}(0)$, the wave functions describing the behaviour of a BPS monopole moving in the field of another BPS monopole, are simply $\mathrm{R}_{\mathrm{nl}}(\mathrm{r}) \mathrm{Y}_{\mathrm{g}, \mathrm{l}, \mathrm{m}}(\theta, \phi)$ with $\mathrm{Y}_{\mathrm{g}, \mathrm{l}, \mathrm{m}}(\theta, \phi)$ as angular function. Thus the Pauli wave function for the spin-up and the spin- down states would be given as

$$
\begin{aligned}
\left(\psi_{+}(0)\right)_{P}= & \psi_{\left(n, l, m_{1}, m_{S}=+1 / 2\right)}=R_{n l} Y_{g, l, m_{1}}|\uparrow\rangle \\
& =\left(\begin{array}{c}
R_{n l} Y_{g, l, m_{1}} \\
0
\end{array}\right)
\end{aligned}
$$

and

$$
\begin{aligned}
\left(\psi_{-}(0)\right)_{P} & =\psi_{\left(n, l, m_{1}, m_{S}=-1 / 2\right.}=R_{n l} Y_{g, l, m_{1}}|\downarrow\rangle \\
& =\left(\begin{array}{c}
0 \\
R_{n l} Y_{g, l, m_{l}}
\end{array}\right) .
\end{aligned}
$$

In the absence of the spin -orbit interaction, both the wave functions correspond to the same energy. We can determine the splitting due to spin-orbit interaction choosing a representation in which $\hat{\mathrm{H}}^{\prime}$ is diagonal

$$
\begin{aligned}
\left(\phi_{1}\right)_{P}= & \phi\left(n, l, j=l+1 / 2, m_{j}\right) \\
& =\sqrt{\frac{l+m_{j}+\frac{1}{2}}{2 l+1}} \psi\left(n, l, m_{l}=m_{j}-\frac{1}{2}, m_{S}=+\frac{1}{2}\right) \\
& +\sqrt{\frac{l-m_{j}+\frac{1}{2}}{2 l+1}} \psi\left(n, l, m_{l}=m_{j}+\frac{1}{2}, m_{S}=-\frac{1}{2}\right)
\end{aligned}
$$

or 


$$
\left(\phi_{1}\right)_{P}=\left(\begin{array}{l}
\sqrt{\frac{l+m_{j}+\frac{1}{2}}{2 l+1}} R_{n l} Y_{g, l, m_{j}-1 / 2} \\
\sqrt{\frac{l-m_{j}+\frac{1}{2}}{2 l+1}} R_{n l} Y_{g, l, m_{j}+1 / 2}
\end{array}\right) .
$$

Then the first order perturbation due to the spin-orbit interaction would be given by

$$
\begin{aligned}
W_{S}^{(1)} & =\int d \tau \phi^{+}\left(\hat{H}^{\prime}\right)_{P} \phi \\
& =\frac{g^{2}}{4 m_{1}^{2} c^{2}} \int d \tau \frac{1}{r^{3}} \phi^{+} 1_{2} \otimes\left[(\hat{J} \otimes \hat{J})_{P}-(\hat{L} \otimes \hat{L})_{P}-(\hat{S} \otimes \hat{S})_{P}\right] \phi
\end{aligned}
$$

or

$$
\begin{array}{r}
W_{S}^{(1)}=\frac{g^{2}}{4 m_{1}^{2} c^{2}}\left[1_{2} \otimes\left\{j(j+1)-l(l+1)-\frac{3}{4}\right\}\right] d \tau \frac{1}{r^{3}}\left\{\frac{l \pm m_{j}+\frac{1}{2}}{2 l+1}\right\} \\
\left|R_{n l}\right|^{2}\left|Y g, l, m_{j}-\frac{1}{2}\right|^{2}+\frac{l \mp m_{j}+\frac{1}{2}}{2 l+1}\left|R_{n l}\right|^{2}|Y| g, l, m_{j}+\left.\frac{1}{2}\right|^{2}
\end{array}
$$

where the plus and minus sign corresponds to $j=l+\frac{1}{2}$ and $j=l-\frac{1}{2}$ respectively. After integration, we get

$$
W_{s}^{(1)}=\left\{\begin{array}{c}
\left.\frac{g^{2}}{4 m_{1}^{2} c^{2}} 1_{2} \otimes l\right) \frac{1}{r^{3}}\langle\text { for } j=l+1 / 2 \\
\left.-\frac{g^{2}}{4 m_{1}^{2} c^{2}} 1_{2} \otimes(l+1)\right\rangle \frac{1}{r^{3}}\langle\text { for } j=l-1 / 2
\end{array}\right.
$$

where

$$
\left\langle\frac{1}{r^{3}}\right\rangle=\int_{0}^{\infty} \frac{1}{r^{3}}\left|R_{n l}\right|^{2} r^{2} d r=\frac{1}{n^{3} l(l+1 / 2)(l+1)} \cdot \frac{1}{a_{0}^{2}} .
$$

The splitting of energy levels corresponding to quantum number $n$ is $W=W^{(0)}+W_{S}^{(1)}$ 


$$
= \begin{cases}E_{n}-\frac{E_{n} g^{2} 1_{2} \otimes l}{2 m_{1}^{2} c^{2} n^{3} l(l+1)(2 l+1) a_{0}^{2}} & \text { for } j=l+1 / 2 \\ E_{n}+\frac{E_{n} g^{2} 1_{2} \otimes(l+1)}{2 m_{1}^{2} c^{2} n^{3} l(l+1)(2 l+1) a_{0}^{2}} & \text { for } j=l-1 / 2\end{cases}
$$

where $E_{n}$ is given by equation (45) and the Bohr radius for the system is given by $a_{0}=\frac{2 \hbar^{2}}{m_{1} g^{2}}$.

Equation (53) gives the splitting of energy levels corresponding to quantum number $n$ for $\mathrm{j}=\mathrm{l}+1 / 2$ and $\mathrm{j}=\mathrm{l}-1 / 2$, respectively.

\section{CONCLUSION}

Equation (10) is Dirac's equation for BPS monopole moving in the field of another BPS monopole in moduli space, which on solving gives Pauli's equation (17) for BPS monopoles. Equation (18) describes the extra spin contribution in the energy gained by BPS monopole while moving in the field of another BPS monopole, which has been interpreted in terms of Bohr magneton and spin moment, through equations (20) and (21) respectively. Equation (28) is the relativistic Hamiltonian, for BPS monopole in the field of another BPS monopole, different parts of which arises due to different relativistic interactions. Hamiltonian of this system has been shown in terms of Higgs potential instead of scalar potential in our recent papers in abelian as well as in non-Abelian gauge theories $^{(14)}$ due to moduli space approximation. Equation (31) is the Schrodinger for a spinning BPS monopole in the field of another BPS monopole in moduli space; spin has been introduced in an adhoc manner in this equation. Equation (45) described the energy eigen value of this system and equations (46) and (47) described the Pauli wavefunction associated with spinning BPS monopoles in moduli space. Equation (53) is splitting of energy levels corresponding to quantum number $n$ and equation (54) is Bohr radius for interacting BPS monopole in moduli space.

\section{REFERENCES}

1. G. 't Hooft, Nucl. Phys. B $\underline{79}, 276$ (1974).

2. A.M. Polyakov, JETP Lett. 20, 194 (1974).

3. E.B. Bogomol'nyi, Sov. J. Nucl. Phys. 24, 449 (1976),

M.K. Prasad and C.M. Sommerfield, Phys. Rev. Lett. $\underline{35}$, 760 (1975). 
4. N.S. Manton and B.J. Schroers, Ann. Phys. 225, 290 (1993).

5. N.S. Manton, Phys. Lett. B 110,54 (1982).

6. G.W. Gibbons and N.S. Manton, Nucl. Phys. B 274, 183 (1986).

7. M.F. Atiyah \& N.J. Hitchin; "The Geometry and Dynamics of Magnetic Monopoles", Princeton Univ. Press, Princeton, NJ, 1988.

8. N. Seiberg, Phys. Rev. D $\underline{49}, 6857$ (1994).

9. R. Jackiw and C. Rebbi, Phys. Rev. D 13 , 3398 (1976).

10. C. Callias, Commun. Math. Phys. 62, 213 (1978).

11. M.P. Singh and B.S. Rajput, Indian J. Phys. 73 A, 425 (1999).

12. M.P. Singh and B.S. Rajput, Pro. Theo. Phys. 102, 843 (1999).

13. C.G. Darwin, Proc. R. Soc. A 118, 634 (1928).

14. P.C. Pant, V.P. Pandey and B.S. Rajput, Il Nuovo Cim. 111A, 1405 (1998).

P.P. Purohit, V.P. Pandey and B.S. Rajput, Indian J. Pure \& Appl. Phys. 38, 287 (2000).

P.P. Purohit, V.P. Pandey and B.S. Rajput, IPSL J. Phys. (2001) (In Press). 